\title{
Variação temporal do Índice de Vegetação por Diferença Normalizada - NDVI no Parque Nacional da Serra da Capivara e seu entorno, Piauí, Brasil
}

\author{
Temporal Variation of The Normalized Difference Vegetation Index (NDVI) at the Serra da \\ Capivara National Park, Piauí, Brazil
}

Aquino $^{1}$, C.M.S; Valladares ${ }^{2}$, G.S.; Aquino ${ }^{3}$, R.P.; Oliveira $^{4}$, J.G.B. cmsaboia@gmail.com

\begin{abstract}
Resumo
O presente trabalho objetivou por meio de imagens do satélite Landsat 5 considerando os anos de 1987 e 2010 analisar a dinâmica do Índice de Vegetação por Diferença Normalizada (NDVI) do Parque Nacional da Serra da Capivara localizado no sudeste do estado do Piauí. Mudanças significativas foram constatadas nas classes de NDVI baixo e solo exposto. Houve incremento na classe baixa de 6,6\% em 1987 para 13,2\%, em 2010, ou seja, esta classe dobrou seu valor. A classe muito baixa aumentou de $85,7 \%$ para $86,6 \%$. O aumento nas classes citadas anteriormente revela a ocorrência de um processo de sucessão ecológica, com aumento do extrato herbáceo na área de estudo. $\mathrm{Na}$ classe solo exposto verificou-se redução de 7,6\% em 1987 para apenas $0,2 \%$ em 2010. De modo geral pode-se afirmar que embora os valores obtidos indiquem baixa atividade fotossintética na área de estudo considerando as imagens utilizadas, houve melhoras na condição do ambiente evidenciado por processos de sucessão ecológica.
\end{abstract}

Palavras-chave: Sensoriamento Remoto, Índice de Vegetação por Diferença Normalizada, Monitoramento.

\begin{abstract}
This work has the objective of analyzing the dynamics of the Normalized Difference Vegetation Index (NDVI) of the Serra da Capivara National Park, localized in the southeastern part of the state of Piauí, through the Landsat 5 satelite images considering the years of 1987 and 2010. Significant changes were evidenced in classes of low NDVI and exposed soil. There was an increment in the low class, variying from $6,6 \%$ in 1987 to $13,2 \%$ in 2010 ; in other words, the class doubled its value. The very low class increased from $85,7 \%$ to $86,6 \%$. The increase in the cited class reveals an occurrence of the process of ecological succession, with an increase of herbaceous extract in the studied area. In the class of exposed soil, a reduction of $7,6 \%$ in 1987 to only $0,2 \%$ in 2010, was verifyed. In general, we can state that inspite of the values obtained indicate low photosynthetic activity in the studied area, considering the utilized images, there was an improvement in the condition of the environment, evidenced by proccesses of ecological succession.
\end{abstract}

Key Words: Remote Sensing, Normalized Difference Vegetation Index, Monitoring.

\section{INTRODUÇÃO}

O sensoriamento remoto é uma ferramenta de obtenção de dados da superfície terrestre, e constitui uma importante técnica para o monitoramento sistemático e dinâmico da vegetação. Utiliza faixas espectrais de bandas no espectro eletromagnético, índices e combinação de bandas, destacando-se para fins de analise da vegetação. Os índices de vegetação resultam de combinações lineares de dados espectrais, realçando o sinal da vegetação, que minimizam as variações na irradiância solar e os efeitos do substrato do dossel vegetal (JACKSON e HUETE, 1991).

\footnotetext{
${ }^{\text {I} C l a ́ u d i a ~ M a r i a ~ S a b o ́ i a ~ d e ~ A q u i n o, ~ C u r s o ~ d e ~ G e o g r a f i a ~ d a ~ U n i v e r s i d a d e ~ F e d e r a l ~ d o ~ P i a u i ́, ~ L a b o r a t o ́ r i o ~ d e ~ G e o m a ́ t i c a, ~ T e r e s i n a, ~ P I, ~}$ Brasil

${ }^{2}$ Gustavo Souza Valladares, Curso de Geografia da Universidade Federal do Piauí, , Laboratório de Geomática, Teresina, PI, Brasil

${ }^{3}$ Renê Pedro de Aquino, Curso de Geografia da Universidade Estadual do Piauí, Teresina, PI, Brasil

${ }^{4}$ José Gerardo Bezerra de Oliveira, Curso de Biologia da Universidade Federal do Ceará, Fortaleza, CE, Brasil
} 
Essa ferramenta desde a década de 1970 assumiu um papel importante no monitoramento e na estimativa dos diversos fenômenos meteorológicos e ambientais, possibilitando a tomada de decisão para fins de preservação ambiental (MOREIRA, 2003).

Os índices de vegetação tem sido muito utilizados no monitoramento de áreas vegetadas, na determinação e na estimativa do índice de área foliar, da biomassa e da radiação fotossinteticamente ativa. (RAMOS, et al. 2010).

Em levantamento realizado Moreira (2003), afirma que podem ser encontrados na literatura mais de 50 índices de vegetação, porém, os mais utilizados são o Razão Simples (RVI) e o Índice de Vegetação por Diferença Normalizada (IVDN), cuja sigla em inglês é NDVI (Normalized Difference Vegetation Index). Segundo Aquino e Oliveira (2012) dentre as metodologias empregadas para identificação e avaliação de mudanças na estrutura, fisionomia e dinâmica da cobertura vegetal entre diferentes datas destaca-se a detecção de mudança espectral a partir do emprego do Índice de Vegetação por diferença Normalizada (NDVI).

Trabalhos como os de Ramos et al. (2010), Rocha et. al (2013) e Nascimento et al. (2013), também utilizaram o NDVI no intuito de identificar diferentes classes de NDVI. Apontaram o método como otimizador no processo de identificação de classes da cobertura vegetal e ainda como sendo o mais sensível à vegetação esparsa, como é o caso da caatinga. Ressalta-se ainda a menor influência de variações atmosféricas quando do emprego deste índice.

Silva et al (2013) avaliou a dinâmica da ocupação do solo e da cobertura vegetal e o NDVI, no município de Parari - PB entre os anos de 1988 e 2005 por meio de técnicas de sensoriamento remoto e analise espacial. Os resultados obtidos com os mapas de cobertura do solo evidenciaram aumento de solo exposto. O solo exposto ocupou em 2005 áreas que em 1988 eram recobertas por vegetação densa e rala. O NDVI representou de forma satisfatória o estado da cobertura vegetal nos anos analisados.

Considerando a comprovada aplicabilidade deste índice o objetivo deste trabalho foi analisar a variação do Índice de Vegetação por Diferença Normalizada (NDVI), nos anos de 190 e 2010 gerado por meio de imagens do satélite LANDSAT 5 TM do Parque Nacional da Serra da Capivara e entorno.

\section{METODOLOGIA}

\section{Processamento digital das imagens}


As imagens do sensor Landsat TM dos anos de 1987 e 2007 foram obtidas gratuitamente junto ao Instituto Nacional de Pesquisas Espaciais (INPE). Os respectivos pontos, órbitas, datas de passagem do satélite, sensor e resolução estão listadas no Quadro 1.

Quadro 1 - Características das imagens Landsat com cobertura de nuvens < 10\% utilizadas para identificação de níveis de NDVI e tipologias de Uso e Cobertura vegetal das terras no Parque Nacional da Serra da Capivara e

Entorno.

\begin{tabular}{|c|c|c|c|c|c|c|c|}
\hline \multicolumn{4}{|c|}{1987} & \multicolumn{4}{|c|}{2010} \\
\hline $\begin{array}{l}\text { Ponto } \\
\text { /órbita }\end{array}$ & Datas & Sensor & Resolução & $\begin{array}{l}\text { Ponto } \\
\text { /órbita }\end{array}$ & Datas & Sensor & Resolução \\
\hline $218 / 66$ & 03/09/1987 & $\begin{array}{c}\text { Landsat } \\
5 \mathrm{TM}\end{array}$ & 30 metros & $218 / 66$ & $04 / 10 / 2010$ & $\begin{array}{c}\text { Landsat } \\
5 \mathrm{TM}\end{array}$ & 30 metros \\
\hline $219 / 66$ & $25 / 08 / 1987$ & $\begin{array}{c}\text { Landsat } \\
5 \mathrm{TM}\end{array}$ & 30 metros & $219 / 66$ & $25 / 09 / 2010$ & $\begin{array}{c}\text { Landsat } \\
5 \mathrm{TM}\end{array}$ & 30 metros \\
\hline
\end{tabular}

As imagens orbitais adquiridas no INPE foram importadas para o software IDRISI 3.2, onde foram georreferenciadas no sistema de coordenadas geográficas (latitude-longitude) a partir do método imagem - imagem através do utilitário RESAMPLE do IDRISI, a partir de imagens ortoretificadas adquiridas junto ao United States Geological Service (USGS - Serviço Geológico dos Estados Unidos), disponível em: http://earthexplorer.usgs.gov utilizando pontos de controle selecionados e distribuidos na área de interesse.

Após o registro das imagens com o uso do utilitário MOSAIC as imagens foram mosaicadas, em seguida com o utilitário Overlay as imagens foram recortadas considerando os limites geográficos da área de estudo, em seguida procedeu-se a reclassificação dos valores obtidos através do aplicativo RECLASS. As classes estabelecidas foram as seguintes: alta, moderadamente alta, moderadamente baixa, baixa, muito baixa e solo exposto. Para o cálculo do NDVI utilizou-se o utilitário VEGINDEX do software IDRISI 3.2 disponível na Universidade Federal do Piauí.

O citado índice possibilita comparações espaciais e temporais da atividade fotossintética terrestre, viabilizando, assim, o monitoramento sazonal, interanual e oscilações de longo prazo dos preceitos estruturais, fenológicos e biofísicos da vegetação (WANG et al., 2003).

Este índice é um modelo resultante da combinação dos níveis de refletância em imagens de satélites, que resulta da equação composta pelas respostas das bandas espectrais do vermelho e 
infravermelho próximo. As refletâncias das bandas 3 (vermelho - visível) e 4 (infravermelho próximo) do sensor LANDSAT 5 TM, resolve-se pela seguinte equação abaixo:

$$
N D V I=\frac{(N I R-R e d)}{(N I R+R e d)}
$$

Onde:

NDVI é o Índice de Vegetação por Diferença Normalizada;

NIR é a refletância no comprimento de onda correspondente ao Infravermelho Próximo $(0,76$ a 0,90 $\mu m$ para o Landsat5);

$\mathrm{R}$ é a refletância no comprimento de onda correspondente ao Vermelho $(0,63$ a $0,69 \mu \mathrm{m}$ para o Landsat5).

Os valores de NDVI computados variam de -1 a +1 . Segundo Almeida et al. (2001) as nuvens, água e neve têm reflectâncias maiores no visível do que no infravermelho, sendo que nestas condições o NDVI tem valores negativos. As rochas e os solos expostos têm reflectâncias similares nas duas bandas e valores próximos a zero; já a vegetação, apresenta maior vigor e densidade, possuindo valores mais altos, permitindo assim a caracterização e a análise da cobertura vegetal.

Nascimento et al (2014) afirmam que o Índice de vegetação por diferença normalizada ou NDVI: são valores que indicam a sensibilidade e a condição da vegetação verde numa determinada área. Os valores variam de -1 a +1 . Em superfícies com vegetação, o NDVI varia de valores próximos a 0 (quase sem vegetação) a 1 (totalmente ou na sua maioria vegetada). Para água e nuvens o NDVI é menor que 0 .

O NDVI analisa a dinâmica da clorofila na faixa do vermelho, onde absorve a radiação solar e resulta em baixa reflectância, no infravermelho próximo a morfologia interna das folhas e a estrutura da vegetação gera alta reflectância da radiação solar incidente (LOURENÇO e LANDIM, 2004). Logo, infere-se que quanto maior for o vigor da vegetação, mais próximo de +1 será o NDVI, bem como maior será o contraste na área imageada.

Rocha et. al (2013) utilizaram o NDVI e apontaram o método como otimizador no processo de identificação de classes da cobertura vegetal. Ramos et. al (2010), utilizaram o NDVI para analisar a degradação no interior do parque Nacional Boqueirão de Onça (PNBO), e apontaram este índice de vegetação como sendo mais sensível a vegetação esparsa, como é o caso da caatinga, afirmativa que justifica a escolha do referido método para a área em estudo, posto a mesma também localizar-se sob domínio de vegetação do tipo caatinga. 


\section{RESULTADOS E DISCUSSÃO}

\section{Dinâmica Interanual do NDVI}

Objetivando avaliar mudanças no NDVI que pudessem evidenciar degradação ambiental na área de estudo, foram estimados e depois comparados os valores deste índice obtidos para os anos de 1987 e 2010, a partir do processo de detecção de mudanças em imagens de satélite que segundo Santos (2005) é definido como o reconhecimento de alterações nos padrões característicos de determinadas feições, em um determinado espaço de tempo.

Os valores de NDVI estimados para a área de estudo foram reclassificados em seis classes: alta, moderadamente alta, moderadamente baixa, baixa, muito baixa e solo exposto, representando os diferentes graus de proteção oferecido pela cobertura vegetal ao solo.

As Figuras 2a e 2b apresentam a espacialização da variabilidade do NDVI na área de estudo. A Tabela 1 apresenta a distribuição absoluta e relativa dos valores de NDVI por classes para os anos considerados.

Ressalta-se que quanto maior for o valor do NDVI, maior será o porte da vegetação. Os índices entre 0 e 0,2 indicam áreas de vegetação quase nula. Entre 0,2 e 0,4 vegetação arbustiva aberta. Entre 0,4 e 0,6, vegetação arbustiva semi-aberta. Os índices entre 0,6 e 0,8 vegetação arbustiva-arbóreo, já os valores entre 0,8 e 1 indicam vegetação arbórea. As áreas de solos expostos foram atribuídos os valores menores que 0 . 


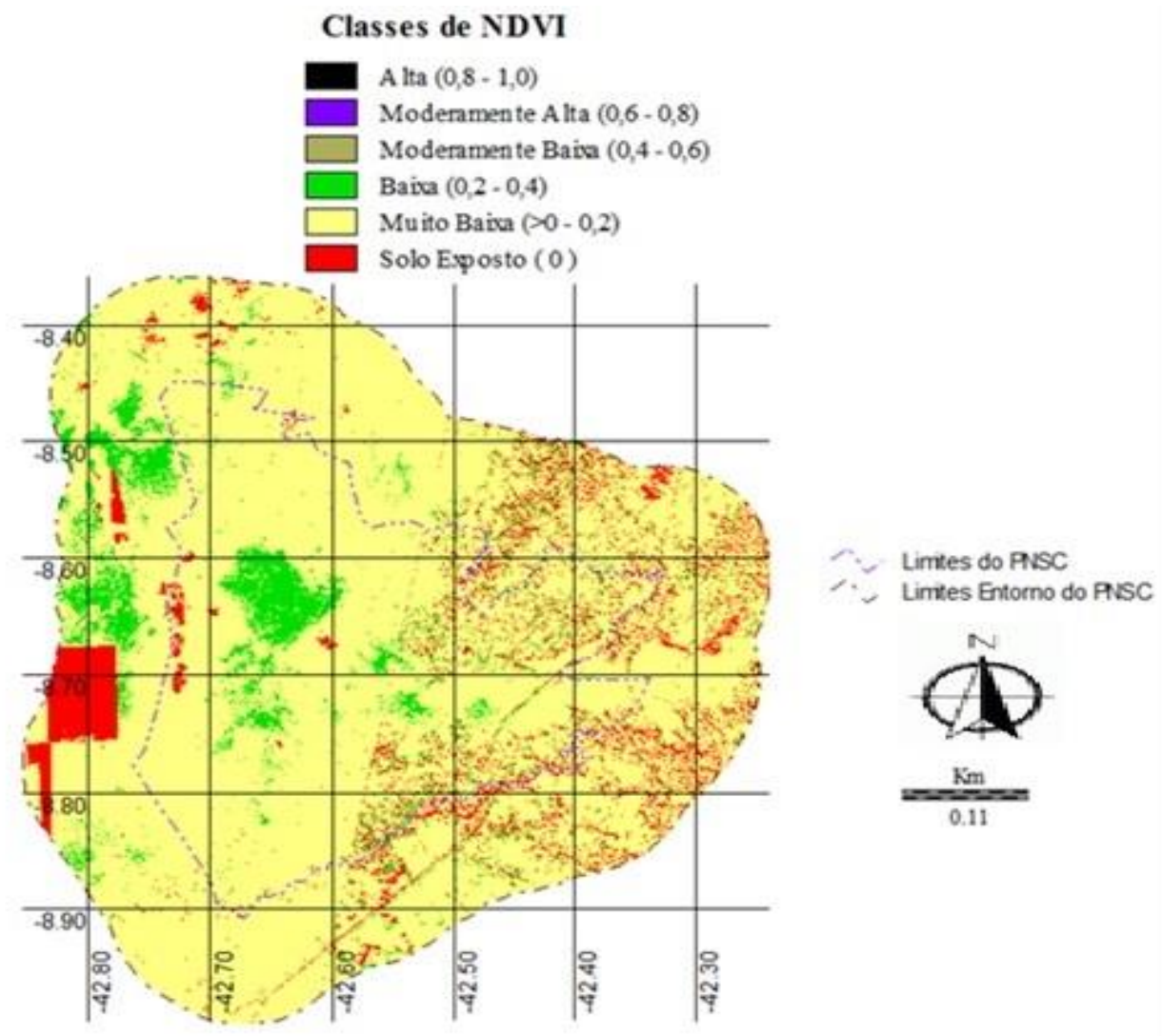

Figura 2a - Índice de Vegetação Normalizado (NDVI) do Parque Nacional da Serra da Capivara e Entorno, 1987.

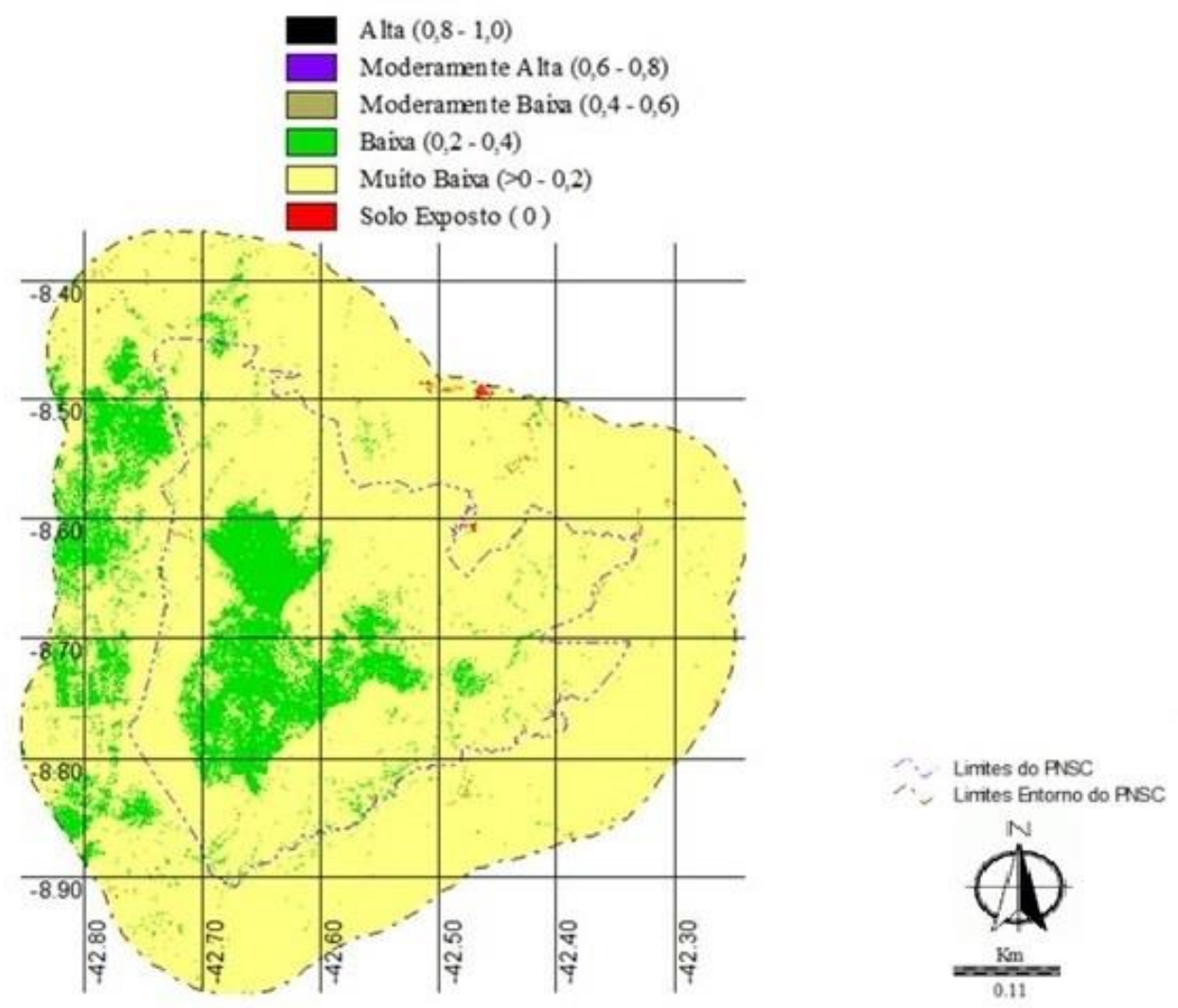


Figura 2b - Índice de Vegetação Normalizado (NDVI) do Parque Nacional da Serra da Capivara e Entorno, 2010.

Tabela 1 - Valores absolutos e relativos de NDVI para o Parque Nacional da Serra da Capivara e Entorno nos anos 1987 e 2010, com as respectivas classes de proteção.

\begin{tabular}{|c|c|c|c|c|c|}
\hline \multirow[t]{2}{*}{$\begin{array}{c}\text { Classes de Proteção } \\
\text { (Faixa de NDVI) }\end{array}$} & \multirow[t]{2}{*}{ Intervalo } & \multicolumn{2}{|c|}{ Área no Nível $\left(\mathrm{km}^{2}\right)$} & \multicolumn{2}{|c|}{$\begin{array}{c}\text { \% da Área Total no } \\
\text { Nível }\end{array}$} \\
\hline & & 1987 & 2010 & 1987 & 2010 \\
\hline (Alta) & 0,8 a 1,0 & 0,0 & 0,0 & 0,0 & 00 \\
\hline $\begin{array}{c}\text { (Moderadamente } \\
\text { alta) }\end{array}$ & $0,6 \mathrm{a}<0,8$ & 0,0 & 0,0 & 0,0 & 00 \\
\hline $\begin{array}{c}\text { (Moderadamente } \\
\text { baixa) }\end{array}$ & $0,4 \mathrm{a}<0,6$ & 1,24 & 0,97 & 0,1 & 0,03 \\
\hline (Baixa) & $0,2 \mathrm{a}<0,4$ & 220,5 & 436,4 & 6,6 & 13,2 \\
\hline (Muito Baixa) & $>0 \mathrm{a}<0,2$ & 2842,8 & 2873,5 & 85,7 & 86,6 \\
\hline Solo Exposto & $\leq 0$ & 252,1 & 6,0 & 7,6 & 0,2 \\
\hline
\end{tabular}

Fonte: os autores

Pela Tabela 1, constata-se não terem sido encontrados valores de NDVI nas classes Alta e Moderadamente Alta na área de estudo, acredita-se devido a época considerada nas imagens, caracterizada pela ausência de chuvas. Mudanças mais significativas foram constatadas nas classes de NDVI baixo e solo exposto. Houve incremento na classe baixa de 6,6\% em 1987 para 13,2\%, em 2010, ou seja, esta classe dobrou seu valor.

A classe muito baixa aumentou de $85,7 \%$ para $86,6 \%$. O aumento nas classes citadas anteriormente revela a ocorrência de um processo de sucessão ecológica, com aumento do extrato herbáceo na área de estudo. Na classe solo exposto verificou-se redução de 7,6\% em 1987 para apenas $0,2 \%$ em 2010. De modo geral pode-se afirmar que embora os valores obtidos indiquem baixa atividade fotossintética na área de estudo considerando as imagens utilizadas, houve melhoras na condição do ambiente evidenciado por processos de sucessão ecológica.

De modo geral, constata-se que na área de estudo a variabilidade interanual do NDVI de 1987 e de 2010 oscila de forma significativa entre as classes muito baixa (> 0 a < 0,2) a baixa $(0,2$ a $<0,4)$. Estes valores são semelhantes aos obtidos em estudo de Braga et al.(2003) que analisando a variabilidade sazonal do NDVI na região Nordeste para o período de 1981 a 1991 constataram que os valores de NDVI oscilaram entre 0,15 e 0,62 . 
Nascimento et al (2014) analisaram o NDVI do município de Cabrobó - PE usando o NDVI e obtiveram oscilando entre 0,1 e 0,6 , valores que estão de acordo com os aqui obtidos, e localizados na mesma região, qual seja o Nordeste brasileiro.

Considerando tratar-se de uma área de preservação, portanto, sem utilização, devido a restrições legais, acredita-se que as variações no NDVI resultem de variação temporal e espacial bastante expressiva em regiões semiáridas a exemplo da área objeto deste estudo.

Vale ressaltar que as variações interanuais de NDVI não necessariamente indicam processos de degradação por antropismo, posto que, fatores abióticos como o clima, têm grande influência sobre a vegetação, fator determinante na atenuação ou aceleração dos processos erosivos. Gurgel (2003) afirma que os valores de NDVI também caracterizam diferenças da cobertura vegetal, resultantes da presença e disponibilidade de recursos naturais distintos. Como por exemplo, corpos d'agua (rios, riachos e córregos) e manchas de solos e afloramentos rochosos.

\section{CONSIDERAÇÕES FINAIS}

O monitoramento com o sensoriamento remoto, por meio dos índices como o NDVI, que tem sido aperfeiçoado e constantemente aplicado na avaliação da vegetação e mostrou adequado no estudo do parque Nacional da Serra da Capivara e seu entorno.

A análise mostrou a ocorrência de um processo de sucessão ecológica evidenciada pelo aumento do extrato herbáceo na área de estudo. Na classe solo exposto verificou-se redução de 7,6\% em 1987 para apenas 0,2\% em 2010. O processo de sucessão ecológica acredita-se resultar da criação do Parque evidenciando a eficácia na criação de unidade de conservação a exemplo do Parque Nacional da Serra da Capivara. Esta constatação permite inferir que o Parque Nacional da Serra da Capivara, cumpre a sua função, qual seja, a manutenção e conservação da biodiversidade do bioma caatinga onde está inserido o referido Parque.

Levantamentos semelhantes a estes devem ser conduzidos nos demais Parque do Estado, posto fornecerem informações necessárias para o monitoramento ambiental especialmente da cobertura vegetal elemento fundamental para a conservação dos recursos hídricos.

\section{REFERÊNCIAS}

ALMEIDA. M.G.; RUDORFF, B.F.T.; SHIMABUKURO, Y.E. Mapeamento da cobertura vegetal de áreas de grande extensão através de mosaicos de imagens do NOAA-AVHRR. In: Anais do X SBSR, Foz do Iguaçu, 21 - 26 abril 2001, INPE, p. 377-381. 
AQUINO, C. M. S. Estudo da degradação / desertificação no núcleo de São Raimundo Nonato

- Piauí. Tese (Doutorado em Geografia). Universidade Federal de Sergipe - UFSE. São Cristovão, 2010.

AQUINO, Claudia Maria Sabóia; OLIVEIRA, José Gerardo Beserra . Estudo da dinâmica do Índice de Vegetacão por Diferença Normalizada (NDVI) no Núcleo de São Raimundo Nonato-PI. GEOUSP - Espaço e Tempo, São Paulo, № 31, pp. 157 - 168, 2012.ISSN: 1414-7416.

BRAGA, C.C.; BRITO, J.I.B.; SANSIGOLO, C.A e RAO; T. V. R. 2003.Tempo de resposta da vegetação às variabilidades sazonais da precipitação no Nordeste do Brasil. Revista Brasileira de Agrometeorologia, Santa Maria-RS, v.11, n.1, p. 149-157.

Brasil. Companhia de Desenvolvimento dos Vales do São Francisco e do Parnaíba - CODEVASF. Plano de Ação para o Desenvolvimento Integrado da Bacia do Parnaíba, PLANAP: síntese executiva : Território dos Cocais / Companhia de Desenvolvimento dos Vales do São Francisco e do Parnaíba - CODEVASF. - Brasília, DF : TDA Desenhos \& Arte Ltda., 2006.

BRASIL. Departamento Nacional de Produção Mineral. Projeto Radam. Levantamento de Recursos Naturais: Parte das Folhas SC.23 - Rio São Francisco e SC.24 Aracaju. Rio de Janeiro, Vol.1, 1973.

CPRM - Companhia de Pesquisa de Recursos Minerais. Ministério de Minas e Energia. Mapa Geológico do Estado do Piauí. $2^{\text {a }}$ Versão. Teresina, 2006

GURGEL, Helen; FERREIRA, Nelson; LUIZ, Alfredo. Estudo da variabilidade do NDVI sobre o Brasil, utilizando-se a análise de agrupamentos. Revista Brasileira de Engenharia Agrícola e Ambiental, Campina Grande, v. 7, nº 1, p. 85-90, 2003. ISSN: 1415-4366.

JACKSON, Ray; HUETE, Alfredo. Interpreting vegetation indices. Preventive Veterinary Medicine,v.11, n.3-4, p.185-200, 1991.

JACOMINE, Paulo Tito. - Levantamento exploratório-reconhecimento de solos do estado do Piauí. Recife: DPP, AgMA/DNPEA, SUDENE/DRN, 1986. (Boletim de pesquisa $\mathrm{n}^{\circ} 26$ ).

LOURENÇO, R. W.; LANDIM, P. M. B. Estudo da variabilidade do "Índice de Vegetação por Diferença Normalizada / NDVI" utilizando krigagem indicativa. HOLOS Environment, v. 4, n. 1. 2004. p.38-55.

MOREIRA, Maurício Alves. Fundamentos do sensoriamento remoto e metodologias de aplicação. 2.ed. Viçosa - MG: UFV, 2003. 307 p. ISBN: 9788572693813

MOREIRA, M.A.; SHIMABUKURO, Y.E. Cálculo do índice de vegetação a partir do sensor AVHRR In: Aplicações ambientais brasileiras dos satélites NOAA e TIROS-N . São Paulo, Oficina de Textos, 2004, cap.4: 79-101 
NASCIMENTO, R.C.; MORAIS, A.C.C.; FEITOSA, H.R.F. Análise espaço-temporal da degradação da caatinga do município de Cabrobó-PE usando NDVI. In: V Simpósio Brasileiro de Ciências Geodésicas e Tecnologias da Geoinformação. Recife - PE, 12- 14 de Nov. de 2014. NASCIMENTO, S. S.; LIMA, Eduardo. Rodrigues Viana.; LIMA, P.P.S. Uso do NDVI na análise temporal da Degradação da Caatinga na Sub-Bacia do Alto Paraíba. OKARA: Geografia em debate, v.8, n.1, 2013, p. 72-93, Paraíba. ISSN: 1982-3878

PELLERIN, J. 1984. Carta geomorfológica da região de São Raimundo Nonato (Piauí), 1984, 1 mapa preto e branco, $48,5 \times 56.5 \mathrm{~cm}$, escala 1.500 .000 .

RAMOS, Ricardo Rivelino Dantas; LOPES, Helio Leandro; MELO JUNIOR, Julio Cesar Ferreira; CANDEIAS, Ana Lucia Bezerra; SIQUEIRA-FILHO, Jose Alves. Aplicação do Índice da Vegetação por Diferença Normalizada (NDVI) na avaliação de áreas degradadas e potenciais para unidades de conservação. III Simpósio Brasileiro de Ciências Geodésicas e Tecnologias da Geoinformação, Recife - PE, de 27 a 30 de Julho de 2010. Recife: Editora da UFPE. Disponível em: https://www.ufpe.br/cgtg/SIMGEOIII/IIISIMGEO_CD/artigos/Todos_Artigos/A_157.pdf.

ROCHA, A. S.; MEIRELES, T.A.V; ANDRADE JÚNIOR, I.O.; Uso do NDVI na Identificação de Classes de Uso do Solo. XIV Seminário de Pesquisa e Pós-Graduação, XII Seminário de Iniciação Científica, IV Seminário PIBID, 25 a 28 de setembro de 2013. Campus Universitário Professor Darcy Ribeiro, Montes Claros-MG. Disponível in: www.fepeg.unimontes.br.

ROUSE, J. W., HAAS, R. H.; SCHELL, J. A.; DEERING, D. W. Monitoring vegetation systems in the great plains with ERTS. In: Earth Resources Technology Satelite-1 Symposium,3,1973. Proceedings. Whashinton,1973,v.1,Sec.A,p.309-317.

SILVA, Glauciene Justino Ferreira da;ALMEIDA, Nadjacleia Vilar; GOMES, Lidiane Cristina Félix; SANTOS, Otávia Karla Apolinário dos. Degradação Ambiental na Microrregião do Carirí Paraibano. Revista Brasileira de Geografia Física,Vol. 6, No 4 (2013). ISSN: 1984-2295.

SANTOS, J. C. 2006. Reconstrução paleoambiental dos depósitos sedimentares neogênicos do Parque Nacional Serra da Capivara e circunvizinhanças, Piauí. Tese de Doutorado. Recife: Centro de Tecnologia e Geociências - Pós Graduação em Geologia - Universidade Federal do Pernambuco - Recife (PE).

SANTOS, G.R. de A.; GUIM, A.; SANTOS, M.V.F.; FERREIRA, M. de A.; LIRA, M. de A.; DUBEUX JÚNIOR,J.C.B.; SILVA M.J. da. Caracterização do pasto de capim-buffel diferido e da dieta de bovinos, durante o período seco no Sertão de Pernambuco. Revista Brasileira de Zootecnia, v.34, p.454-463, 2005. 
SARTORI, A.A. C. Análise multitemporal do uso e cobertura do solo na Área de Proteção Ambiental (APA) Tejupá, no período de 1984 a 2011. Anais XVI Simpósio Brasileiro de Sensoriamento Remoto - SBSR, Foz do Iguaçu, PR, Brasil, p.7048-7055. 13 - 18 abr. 2013.

THULER, L.H. M, Org. Estudo da Dinâmica Florestal no Parque Estadual Cachoeira da Fumaça por meio de Subtração de Imagens NDVI. Enciclopédia Biosfera, Centro Científico Conhecer Goiânia, v.8, N.14; p. 1251-1262, 2012. ISSN: 1809-0583.

USGS - United States Geological Service (Serviço Geológico dos Estados Unidos). Earth Explorer - Collection - Landsat Archive. Disponível em <http://earthexplorer.usgs.gov>. Acesso em 27 de novembro de 2015.

WANG, Z. X.; LIU, C.; HUETE, A. From AVHRRNDVI to MODIS4EVI: Advances in vegetation index research.Acta Ecologica Sinica, v.23, n.5, p.979-988, 2003.

\section{AGRADECIMENTO}

Os autores agradecem a Fundação de Amparo a Pesquisa do Estado do Piauí pelo recurso financeiro para o desenvolvimento deste trabalho, que integra um projeto maior (PPP EDITAL 004/2011).

Recebido em: 14/08/2016

Aceito para publicação em: 01/10/2016 